\title{
Vehicle routing optimization with multiple fuzzy time windows based on improved wolf pack algorithm
}

\author{
Cao, Q.K. ${ }^{a}$, Yang, K.W. ${ }^{a}$, Ren, X.Y. ${ }^{a,}{ }^{*}$

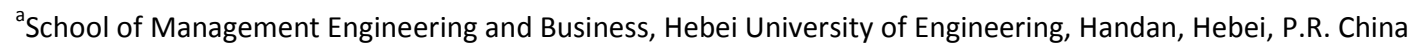

\begin{abstract}
A B S T R A C T
The vehicle routing problem with multiple fuzzy time windows is investigated in this paper. The dynamic change of traffic flow and the fuzzy time window of customers are considered. A multi fuzzy time window vehicle routing model based on time-varying traffic flow is proposed, and the objective function is to minimize the total cost of distribution and maximize customer satisfaction. According to the basic principle of wolf pack algorithm, in order to promote the exchange of information between the artificial wolves, improve the wolves' grasp of the global information and enhance the exploring ability of wolves, a drift operator and wave operator were introduced into scouting behaviors and summing behaviors. An adaptive dynamic adjustment factor strategy was proposed for beleaguering behaviors, the exploitation ability of the algorithm strengthened constantly. Thus the convergence rate of algorithm was enhanced. We further do simulation on an example, and compare the results obtained by wolf pack algorithm and ant genetic algorithm. The results show that use improved wolf pack algorithm to solve vehicle routing problem with multiple fuzzy time windows has the advantages of small number of iterations and high efficiency, it can converge to the global optimal solution in a short time. The improved wolf pack algorithm is an efficient algorithm for solving vehicle routing problem with multiple fuzzy time windows.
\end{abstract}

\section{ARTICLE INFO}

Keywords:

Vehicle routing

Traffic flow

Multi fuzzy time windows

Wolf pack algorithm

Customer satisfaction

*Corresponding author: boyrenxy@126.com

(Ren, X.Y.)

Article history:

Received 3 July 2017

Revised 12 October 2017

Accepted 8 November 2017

(c) 2017 PEI, University of Maribor. All rights reserved.

\section{References}

[1] Dantzig, G.B., Ramser, J.H. (1959). The truck dispatching problem, Management Science, Vol. 6, No. 1, 80-91, doi: $\underline{10.1287 / \mathrm{mnsc} .6 .1 .80 \text {. }}$

[2] Lu, X.C., Chen, Q.B., Zhang, Z.J. (2014). The electric vehicle routing optimizing algorithm and the charging stations' layout analysis in Beijing, International Journal of Simulation Modelling, Vol. 13, No. 1, 116-127, doi: 10.2507/ IJSIMM13(1)CO4.

[3] Cao, Q.-K., Liu, X.-Y., Ren, X.-Y. (2015). Vehicle scheduling problem based on plant growth simulation algorithm, Systems Engineering - Theory \& Practice, Vol. 35, No. 6, 1449-1456, doi: 123.57.41.99/jweb xtgcllysj/CN/ 10.12011/1000-6788(2015)6-1449.

[4] Wang, X., Ruan, J., Sun, Z., Cao, H. (2013). Disruption recovery model for vehicle routing problem with backhaul, Journal of Systems Engineering, Vol. 28, No. 5, 608-616.

[5] Cordeau, J.-F., Laporte, G., Mercier, A. (2001). A unified tabu search heuristics for vehicle routing problems with time windows, Journal of the Operational Research Society, Vol. 52, No. 8, 928-936, doi: 10.1057/palgrave.jors. $\underline{2601163}$.

[6] Qureshi, A.G., Taniguchi, E., Yamada, T. (2009). An exact solution approach for vehicle routing and scheduling problems with soft time windows, Transportation Research Part E: Logistics \& Transportation Review, Vol. 45, No. 6, 960-977, doi: org/10.1016/j.tre.2009.04.007.

[7] Hong, L. (2012). An improved LNS algorithm for real-time vehicle routing problem with time windows, Computers \& Operations Research, Vol. 39, No. 2, 151-163, doi: org/10.1016/i.cor.2011.03.006. 
[8] He, X.-F., Ma, L. (2013). Quantum-inspired ant colony algorithm for vehicle routing problem with time windows, Systems Engineering - Theory \& Practice, Vol. 33, No. 5, 1255-1261, doi: 10.3969/j.issn.1000-6788.2013.05.021.

[9] Meng, X.-H., Hu, R., Qian, B. (2014). Effective hybrid population-based incremental learning algorithm for vehicle routing problem with time windows, Systems Engineering - Theory \& Practice, Vol. 34, No. 10, 2701-2709, doi: 10.12011/1000-6788(2014)10-2701.

[10] Shao, J.-P., Cao, Q., Shen, M., Sun, Y. (2015). Research on multi-objective optimization for fresh agricultural products VRP problem, Industrial Engineering and Management, Vol. 20, No. 1, 122-127, doi: 10.3969/j.issn.10075429.2015.01.019.

[11] Li, Z.P., Zhao, F., Liu, H.D. (2015). Intelligent water drops algorithm for vehicle routing problem with multiple time windows, Operations Research and Management Science, Vol. 24, No. 6, 1-10.

[12] Yan, F., Wang, Y. (2016). Modeling and solution of vehicle routing problem with multiple fuzzy time windows, Journal of Transportation Systems Engineering and Information Technology, Vol. 16, No. 6, 182-188, doi: 10.3969/j.issn.1009-6744.2016.06.028.

[13] Van Woensel, T., Kerbache, L., Peremans, H., Vandaele, N. (2008). Vehicle routing with dynamic travel times: A queueing approach, European Journal of Operational Research, Vol. 186, No. 3, 990-1007, doi: 10.1016/i.ejor. 2007.03.012.

[14] Kritzinger, S., Doerner, K.F., Hartl, R.F., Kiechle, G. Y., Stadler, H., Manohar, S.S. (2012). Using traffic information for time-dependent vehicle routing, Procedia - Social and Behavioral Sciences, Vol. 39, 217-229, doi: 10.1016/ j.sbspro.2012.03.103.

[15] Kok, A.L., Hans, E.W., Schutten, J.M.J. (2012). Vehicle routing under time-dependent travel times: The impact of congestion avoidance, Computers \& Operations Research, Vol. 39, No. 5, 910-918, doi: 10.1016/i.cor.2011. 05.027.

[16] Li, Y., Li, J., Gao, Z. (2012). Dynamic programming heuristics for solving time dependent vehicle routing problem, Systems Engineering - Theory \& Practice, Vol. 32, No. 8, 1712-1718, doi: 10.3969/i.issn.1000-6788.2012.08.010.

[17] Tagmouti, M., Gendreau, M., Potvin, J.-Y. (2007). Arc routing problems with time-dependent service costs, European Journal of Operational Research, Vol. 181, No. 1, 30-39, doi: 10.1016/j.ejor.2006.06.028.

[18] Van Woensel, T., Kerbache, L., Peremans, H., Vandaele, N. (2008). Vehicle routing with dynamic travel times: a queueing approach, European Journal of Operational Research, Vol. 186, No. 3, 990-1007, doi: 10.1016/i.ejor. 2007.03.012.

[19] Donati, A.V., Montemanni, R., Casagrande, N., Rizzoli, A.E., Gambardella, L.M. (2008). Time dependent vehicle routing problem with a multi ant colony system, European Journal of Operational Research, Vol. 185, No. 3, 11741191, doi: 10.1016/j.ejor.2006.06.047.

[20] Xiang, Z., Chu, C., Chen, H. (2008). The study of a dynamic dial-a-ride problem under time-dependent and stochastic environments, European Journal of Operational Research, Vol. 185, No. 2, 534-551, doi: 10.1016/i.ejor. 2007.01.007.

[21] Shi, Z., Fu, Z. (2013). Distribution location routing optimization problem of food cold chain with time window in time varying network, Application Research of Computers, Vol. 30, No. 1, 183-188, doi: 10.3969/j.issn.10013695.2013.01.047.

[22] Zhu, T., Wang, X.L., Zhao, L.J. (2016). Path selection of hazardous materials road transportation with time window and multi-objectives, Industrial Engineering Journal, Vol. 19, No. 2, 62-67, doi: 10.3969/j.issn.1007-7375. 2016.02.010.

[23] Wu, H.S., Zhang, F.M., Wu, L.S. (2013). New swarm intelligence algorithm-wolf pack algorithm, Systems Engineering and Electronics, Vol. 35, No. 11, 2430-2438.

[24] Wu, H.-S., Zhang, F.-M., Li, H., Liang, X.-L. (2015). Discrete wolf pack algorithm for traveling salesman problem, Control and Decision, Vol. 30, No. 10, 1861-1867, doi: 10.13195/j.kzyic.2014.1055.

[25] Ye, Y., Zhang, H.Z. (2017). Wolf pack algorithm for multi-depot vehicle routing problem, Application Research of Computers, Vol. 34, No. 9, 2590-2593.

[26] Li, G., Wei, Z., Xu, L. (2015). Wolf pack algorithm based on modified search strategy, Journal of Computer Applications, Vol. 35, No. 6, 1633-1636, doi: 10.11772/j.issn.1001-9081.2015.06.1633. 


\title{
Optimizacija usmerjanja vozil z več mehkimi časovnimi okni s pomočjo izboljšanega algoritma krdela volkov
}

\author{
Cao, Q.K. ${ }^{a}$, Yang, K.W. ${ }^{a}$, Ren, X.Y. ${ }^{a,}$ \\ ${ }^{\text {a }}$ School of Management Engineering and Business, Hebei University of Engineering, Handan, Hebei, P.R. China
}

\section{POVZETEK}

V prispevku je obravnavan problem usmerjanja vozil z več mehkimi časovnimi okni. Upoštevano je dinamično spreminjanje prometnega toka in mehko časovno okno strank. Predlagan je večfazni model usmerjanja vozil, ki temelji na spreminjajočem se prometnem toku. Ciljna funkcija je zmanjšati skupne stroške distribucije in povečati zadovoljstvo kupcev. Uporabljen je izboljšan algoritem krdela volkov. Da bi spodbudili izmenjavo informacij med umetnimi volkovi, izboljšali razumevanje globalnih informacij o volkovih in povečali njihovo sposobnost raziskovanja, smo v osnovni algoritem krdela volkov v mehanizem raziskovanja in združevanja volkov dodali drsni in valovni operator. Vpeljan je bil tudi faktor dinamičnega prilagajanja, ki krepi eksplotacijske zmožnosti algoritma in izboljša njegovo konvergenco. Izvedena je bila tudi primerjava med izboljšanim algoritmom krdela volkov in genetskim algoritmom. Rezultati kažejo, da ima uporaba izboljšanega algoritma krdela volkov za reševanje problema usmerjanja vozil z več mehkimi časovnimi okni prednosti v smislu majhnega števila iteracij in visoke učinkovitosti in lahko v kratkem času konvergira v globalno optimalno rešitev. Izboljšani algoritem krdela volkov je učinkovit algoritem za reševanje problemov izbire poti $\mathrm{z}$ več mehkimi časovnimi okni.

\section{PODATKI O ČLANKU}

Ključne besede:

Usmerjanje vozil

Prometni tok

Več mehkih časovnih oken

Algoritem krdela volkov

Zadovoljstvo kupcev

*Kontaktna oseba: boyrenxy@126.com (Ren, X.Y.)

Zgodovina članka: Prejet 3. julija 2017

Popravljen 12. oktobra 2017

Sprejet 8. novembra 2017 